\title{
On the asymmetry of Gamow-Teller $\beta$-decay rates in mirror nuclei in relation with second-class currents
}

\author{
N. A. Smirnova ${ }^{1,2}$, C. Volpe ${ }^{3,4}$ \\ 1 Instituut voor Kern- en Stralingsfysica, University of Leuven, Celestijnenlaan 200 D, 3001 Leuven, Belgium \\ 2 University of Ghent, Institute for Nuclear Science, Proeftuinstraat 86, \\ B-9000 Ghent, Belgium; E-mail: nadya.smirnova@rug.ac.be \\ 3 Institut für Theoretische Physik, Universität Heidelberg, Philosophenweg 19, \\ D-69120 Heidelberg, Germany; E-mail: volpe@tphys.uni-heidelberg.de and \\ 4 Institut de Physique Nucléaire, Bât. 100, F 91406, Orsay cedex, France
}

(Dated: October 29, 2018)

\begin{abstract}
The theoretical evaluation of major nuclear structure effects on the asymmetry of allowed GamowTeller $\beta$-decay rates in light mirror nuclei is presented. The calculations are performed within the shell model, using empirical isospin-nonconserving interaction and realistic Woods-Saxon radial wave functions. The revised treatment of $p$-shell nuclei is supplemented by systematic calculations for $s d-$ shell nuclei and compared to experimental asymmetries when available. The results are important in connection with the possible existence of second-class currents in the weak interaction.
\end{abstract}

PACS numbers: 23.40.Hc,21.60.Cs,12.15.-y

Corresponding author:

Nadya A. Smirnova

University of Ghent, Institute for Nuclear Science,

Proeftuinstraat 86, B-9000 Ghent, Belgium

Tel: $(+32)(0) 92646541$

Fax: (+32) (0)9 2646697

E-mail: nadya.smirnova@rug.ac.be

\section{INTRODUCTION}

One of the possibilities to look for the existence of second-class currents in the weak interaction is through the study of differences in allowed Gamow-Teller (GT) $\beta$-decay rates of mirror nuclei [1, 2, 3, 4, 5].

Weak processes in nuclei at low energy are well described by the effective $\mathcal{V}-\mathcal{A}$ interaction :

$$
H_{\mathcal{V}-\mathcal{A}}=\frac{G_{F}}{\sqrt{2}} J_{\mu}^{\dagger} j^{\mu}+\text { h.c. }
$$

where $J_{\mu}^{\dagger}\left(j^{\mu}\right)$ is the hadronic (leptonic) current, and $G_{F}$ is the weak interaction coupling constant. The most general Lorentz covariant form for the hadronic current reads $J_{\mu}^{\dagger}=V_{\mu}+A_{\mu}$ with

$$
\begin{aligned}
V_{\mu} & =i \bar{\psi}_{p}\left[g_{V} \gamma_{\mu}+\frac{g_{M}}{2 M} \sigma_{\mu \nu} k_{\nu}+i g_{S} k_{\mu}\right] \psi_{n}, \\
A_{\mu} & =i \bar{\psi}_{p}\left[g_{A} \gamma_{\mu} \gamma_{5}+\frac{g_{T}}{2 M} \sigma_{\mu \nu} \gamma_{5} k_{\nu}+i g_{P} k_{\mu} \gamma_{5}\right] \psi_{n}
\end{aligned}
$$

standing for the vector and axial-vector parts. Here $k_{\mu}$ is the momentum transferred, $M$ is the nucleon mass, $\psi_{p}$ $\left(\psi_{n}\right)$ is the proton (neutron) field operator. The six terms are the main vector, weak magnetism, induced scalar in (2) and the main axial-vector, induced tensor and induced pseudoscalar in (3), with $g_{V}, g_{M}, g_{S}, g_{A}, g_{T}, g_{P}$ being the corresponding coupling constants.

The weak hadronic currents in Eqs. (2)-(3) can be classified 1] according to their transformation properties under the $\mathcal{G}$-parity operation, $\mathcal{G}=\mathcal{C} \exp \left(i \pi \mathcal{T}_{2}\right)$, i.e. the product of charge conjugation $(C)$ and rotation over $180^{\circ}$ about the 2 -axis in isospin space. Under this transformation, the main vector term, weak magnetism in $V_{\mu}$ (2) and the main axial-vector and induced pseudoscalar terms in $A_{\mu}(\underline{3})$ are even $\left(\mathcal{G} V_{\mu}^{I} \mathcal{G}^{\dagger}=V_{\mu}^{I}\right)$ and odd $\left(\mathcal{G} A_{\mu}^{I} \mathcal{G}^{\dagger}=-A_{\mu}^{I}\right)$, respectively. They are usually called first-class currents. The induced scalar term in $V_{\mu}$ and induced tensor in $A_{\mu}$ transform, under $\mathcal{G}$, with opposite sign with respect to first-class currents. They are usually referred to as second-class currents $(s c c)$. 
The interest in the related weak form factors stem both from the desire of understanding them in the framework on quantum chromodynamics and in the search for the physics beyond the Standard Model [ 6 ].

Constraints on weak form factors come from two hypothesis that follow from approximate symmetries of the Standard Model : the conserved vector current (CVC) hypothesis and the partially conserved axial-vector current (PCAC) hypothesis. In the limit of exact isospin symmetry, the CVC hypothesis ensures that : i) the weak form factors $g_{V}\left(k^{2}\right)$ and $g_{M}\left(k^{2}\right)$ are related to the electromagnetic ones; ii) the induced scalar form factor $g_{S}(k)$ vanishes. Therefore, the latter term can contribute only at the level of isospin symmetry breaking.

The PCAC hypothesis does not put any limits on the value of $s c c$ induced tensor form factor $g_{T}\left(k^{2}\right)$. Thus, the nonconservation of the $\mathcal{G}$ symmetry (due for example to differences in $u$ and $d$ quark masses and to the electromagnetic interaction) will result in a non-zero contribution of $g_{T}$.

It is an experimental and theoretical challenge to place limits on the possible presence of the induced tensor term in the axial-vector current. The search for evidence of its existence is longstanding [4, 5].

In particle physics, the evidence can be obtained, for example, from $G$-parity non-conserving decay branch, such as $\tau \rightarrow \eta \pi \nu$ [7], or from the angular distribution analysis after the decay $\tau \rightarrow \omega \pi \nu$ involving both first and second-class currents [8].

The study of weak processes in nuclei offers various possibilities in this search. There are two limits on the value of $g_{T}$ obtained from muon capture experiments, which are consistent with the absence of second-class currents $[9$, , 10].

In $\beta$ decay, one can determine possible contributions from the induced tensor term in two types of measurements [4]. The first one is through correlation experiments where one measures the correlation between nuclear spin and momentum of emitted $\beta$-particle in the decay of oriented systems, or the correlation between the momentum of the emitted $\beta$-particle and of the radiation from a daughter state. A recent re-measurement of $\beta$-particles angular distribution in the decay of aligned ${ }^{12} \mathrm{~B}$ and ${ }^{12} \mathrm{~N}$ gives a non-zero value of $g_{T}$ 11. These experiments require the knowledge of the weak magnetism coupling constant, which gives another uncertainty. Only in the last years an independent way to extract $s c c$, based on the $\beta$ - $\alpha$ angular correlation in $A=8$ system, has been proposed [12, 13].

Besides, the second-class currents can give rise to different rates of mirror $\beta_{ \pm}$-decay transitions [1]. This phenomenon was actively explored in early seventies, stimulated by first experimental observations of large asymmetries in the $f t$ values in the decay of light nuclei [14]. The experimental asymmetry deduced from measured halflives

$$
\delta=\frac{(f t)_{+}}{(f t)_{-}}-1
$$

where $(f t)_{ \pm}$refer to the $\beta_{ \pm}$decays in the mirror nuclei, is known at present to vary on average from $1 \%$ to several tens of percent, being about $(5 \pm 4) \%$ on average [15]. From the theoretical point of view, the asymmetry $\delta$ (4) can have two origins : i) the possible existence of the scc term in the axial-vector current, i.e. non-zero contribution of $g_{T}\left(\delta^{s c c}\right)$; ii) the breaking of the only approximate isospin symmetry between the decaying nuclear states $\left(\delta^{n u c l}\right)$, i.e.

$$
\delta=\delta^{s c c}+\delta^{n u c l} .
$$

Assuming the perfect mirror symmetry for the nuclear states and impulse approximation, the induced tensor current gives rise [2] to the asymmetry $\delta^{s c c}$ proportional to the induced tensor constant and the sum of maximum energy releases in both mirror transitions, $\left(W_{0}^{+}+W_{0}^{-}\right)$. The more refined calculations [3], including the off mass-shell effects and meson exchange corrections which are crucial in many-body physics [16], predict the asymmetry provided by the second-class currents to be

$$
\delta^{s c c}=-4 \frac{\lambda}{g_{A}} J+\frac{2}{3 g_{A}}(\lambda L-2 \zeta)\left(W_{0}^{+}+W_{0}^{-}\right) .
$$

Here $\lambda$ and $\zeta$ are two parameters related to the coupling constants for off-shell and mesonic corrections, and induced tensor coupling constants, $J$ and $L$ are ratios of the matrix elements of meson-exchange currents between nuclear states (see Ref. [3] for more details).

However, it has been shown [17, 18, 19, 20], that the nuclear structure effects provide the principal contribution to the asymmetry, from $10 \%$ to $20 \%$ for different nuclei, making the extraction of the possible contribution from induced tensor term an extremely difficult task $[3,5]$.

All early estimations [17, 18, 19, 20] of $\delta^{\text {nucl }}$ were carried out within the nuclear shell model for a few $p$-shell nuclei only (namely, for $A=8,9,12,13$ ), since the proper treatment of the $s d$-shell nuclei was far outside available at that time computational power. The basic ingredients of the calculations were the Cohen-Kurath interaction [21] with empirical isospin-nonconserving (INC) corrections [20] and the radial wave functions obtained with the Woods-Saxon (WS) potential. The results obtained have been used to extract limits on second-class currents [3, 5].

To be precise, the main contribution to $\delta^{\text {nucl }}$ comes from the difference in the matrix elements of the GT operator $(\sigma \tau)$ between nuclear states, due to isospin mixing and differences in the radial wave functions, as we extensively 
discuss below. The calculations of resulting asymmetry in $f t$-values performed by different authors [17, 18, 19, 20] vary significantly, either because of different choices in the parametrizations or because of the more and more refined shell model technique used by the subsequent authors.

Apart from this, there are some contributions to $\delta^{\text {nucl }}$ due to the higher order effects, most of which are usually incorporated as corrections to the statistical rate function $f$, i.e. forbidden matrix elements, known induced currents, such as the induced pseudoscalar (see Ref. 2] for a complete list). Contrary to the calculation of the main part of $\delta$, the evaluation of higher order corrections, using different nuclear wave functions [17, 20], gives quite similar values and in any case does not exceed a few percent.

Since the mentioned above estimations of the GT transition rates in mirror nuclei, no other attempts to refine the calculations have been undertaken up to now, in spite of the essential improvement in the knowledge of the low-energy nuclear structure. Wider and more accurate experimental data on $\beta$ decay [15] are available nowadays, including $s d$-shell nuclei. In Fig. 1 we show the asymmetry extracted from the measured $\beta$-decay lifetimes of the selected mirror transitions for most of which the asymmetry is different from zero by $2 \sigma$ or more.

Besides, the theoretical description of $p$-shell and $s d$-shell nuclei has made much progress during the last thirty years 22]. Since the limits on the induced tensor current are still not settled [5, 6, [9, 10, 11], it is worthwhile to improve the constraints on second-class currents coming from the $\beta$ decay of nuclei using on one hand the current experimental systematics and on the other hand improved calculations.

The aim of this paper is to present up-to-date shell model calculations, for $p$-shell and, for the first time, $s d$-shell nuclei, of the main nuclear structure effects on the asymmetry $\delta^{n u c l}$ (5) arising from the difference of GT operator matrix elements due to isospin mixing in nuclear states and differences in the radial wave functions. The higher order corrections can present a subject for a future work. In this study, we use $p$-shell [21] and $s d$-shell model wave functions [23] obtained from the INC Hamiltonians [24], in a sufficiently large model space easily accessible now, and realistic radial dependence, and we compare the results with the experimental data.

The theoretical framework, we choose, follows the one of Towner [20] and goes beyond it in several respects. First, we use the better fitted INC interaction to take into account isospin breaking, both without and within the parent state formalism, similarly to the calculations of Ormand and Brown 25] for superallowed transitions. Second, we use a different parametrization of the WS potential. We carefully investigate the sensitivity of the asymmetry to the way the tail of the single-particle wave functions may be determined by fitting the single-particle energies to the experimental separation energies. In fact, there are two possible procedures : I) to adjust the energy of the last unoccupied single-particle state only, as in Ref. 19, 20]; II) to fix all the energies of the single-particle states in the considered shell at the experimental separation energy, as it is done in Ref. [18] for $A=12$ only. Strictly speaking, none of them is exact. However, we will argue that the latter is more consistent with the shell model, while the former can even lead to "unrealistic" results when the last unoccupied single-particle state is unbound. We will then use, contrary to previous calculations [19, 20], the procedure of fitting all the single-particle energies at the same experimental separation energy to estimate the asymmetry for an ensemble of $p$ - and $s d$-shell nuclei. We fit the separation energy either by adding a surface term to the WS potential or by varying the volume term, as it is done in Ref. [18, 19, 20].

In previous works on asymmetries and second-class currents [17, 18, 19, 20], it was never stressed that one should discuss the $B(\mathrm{GT})$-values before considering the corresponding asymmetries. Here we carefully examine the $B(\mathrm{GT})$ values and test the wave functions by comparing certain electromagnetic observables, namely magnetic and quadrupole moments, to available experimental data. The problem of the effective GT transition operator in relation with the quenching of the GT strength is also discussed.

We remark that to extract limits on second-class currents in $\beta$ decay one needs calculations on $B(\mathrm{GT})$-values as precise as possible and therefore should concentrate on the best described nuclei. In the context of the shell model, it means that very light nuclei, possibly viewed as few-nucleon systems, may in principle not be very good candidates. For example, the lightest nuclei, such as those involved in the $A=8,9$ cases, should be treated with particular techniques. This is also why, contrary to what was done in the past, in the present work we make an extensive analysis of both $p$-shell and $s d$-shell nuclei (Fig. 1).

In the present study we restrict ourselves to the WS wave functions. As was noticed in Ref. [25], the WS potential may overestimate the difference in the tails of proton and neutron radial wave functions due to differences in the Coulomb and isovector potentials. The results shown in 25] indicate that calculations with the wave functions obtained from the Hartree-Fock procedure would lead to a smaller effect on the asymmetry. However, as we will show, the present level of accuracy on the $B(\mathrm{GT})$-values is still not good enough for extracting possible contributions of the second-class currents from the asymmetries. In this respect, calculations with Hartree-Fock wave functions would not improve the $B(\mathrm{GT})$ estimates enough to change these conclusions. For these reasons, we present results obtained with WS wave functions only which provide the upper limit of theoretical asymmetries.

The paper is organized as follows. In Section II we describe the theoretical approach, we give the details of the interaction used, the parameters of the single-particle WS potential and discuss the different procedures. Section 
III is devoted to the presentation of the results on magnetic and quadrupole moments, transition probabilities and asymmetries obtained for $p$ - and $s d$-shell nuclei. Summary and conclusions are given in the last section.

\section{THEORETICAL FRAMEWORK}

Within the nuclear shell model [26], the asymmetry (4) is related to the GT matrix elements $M_{ \pm}$for $\beta_{+}$and $\beta_{-}$ decay through

$$
\delta=\left|\frac{M_{-}}{M_{+}}\right|^{2}-1
$$

with

$$
\begin{aligned}
M_{ \pm} \equiv & \left\langle f\left\|\sum_{k=1}^{n} \hat{\sigma}(k) \hat{\tau}_{ \pm}(k)\right\| i\right\rangle= \\
= & \sum_{j_{1}, j_{2}, \pi}(-1)^{J_{f}+J_{\pi}+j_{2}+1} \sqrt{\left(2 J_{i}+1\right)\left(2 J_{f}+1\right)}\left\{\begin{array}{ccc}
J_{f} & J_{i} & 1 \\
j_{1} & j_{2} & J_{\pi}
\end{array}\right\} \\
& S^{1 / 2}\left(j_{2} ; J_{f} J_{\pi}\right) S^{1 / 2}\left(j_{1} ; J_{i} J_{\pi}\right)\left\langle n_{2} l_{2} j_{2}\left\|\hat{\sigma} \hat{\tau}_{ \pm}\right\| n_{1} l_{1} j_{1}\right\rangle,
\end{aligned}
$$

where $n$ is the number of valence particles in the many-body wave functions that describe the initial, $|i\rangle$, and final, $|f\rangle$, states in mother and daughter nuclei with angular momenta $J_{i}$ and $J_{f}$ respectively; $J_{\pi}$ is the angular momentum of the parent states $\pi$ of the $(A-1)$ nucleus and $S^{1 / 2}\left(j ; J J_{\pi}\right)$ denotes a spectroscopic amplitude with a proper phase factor, and gives the mixing of different shell model configurations in the eigenfunction. The INC terms in the effective shell-model interaction are responsible for the difference in the values of $S^{1 / 2}\left(j ; J J_{\pi}\right)$ for mirror transitions. The single-nucleon reduced matrix element is defined as

$$
\left\langle n_{2} l_{2} j_{2}\left\|\hat{\sigma} \hat{\tau}_{ \pm}\right\| n_{1} l_{1} j_{1}\right\rangle=\delta_{l_{1} l_{2}}(-1)^{j_{1}+l_{1}+3 / 2} \sqrt{6} \sqrt{\left(2 j_{1}+1\right)\left(2 j_{2}+1\right)}\left\{\begin{array}{ccc}
1 / 2 & j_{2} & l_{1} \\
j_{1} & 1 / 2 & 1
\end{array}\right\} \Omega_{j_{1} j_{2}}^{\pi}
$$

with $\left(n_{i}, l_{i}, j_{i}\right)$ labelling the single-particle states in a spherical basis and $\Omega_{j_{1} j_{2}}^{\pi}$ being the overlaps of the single-nucleon radial wave functions :

$$
\Omega_{j_{1} j_{2}}^{\pi}=\int_{0}^{\infty} R_{n_{1} l_{1} j_{1}}^{\pi}(r) R_{n_{2} l_{2} j_{2}}^{\pi}(r) r^{2} d r
$$

The radial single-particle wave functions $R_{n_{1} l_{1} j_{1}}^{\pi}(r)$ are obtained from a spherically symmetric single-particle potential. For realistic radial wave functions, obtained from WS or Hartree-Fock potential with Coulomb and other chargedependent corrections, the integrals (10) are non-identical for two mirror processes. The difference in the matrix elements of GT operator for $\beta_{+}$and $\beta_{-}$-decays (8) is extremely sensitive to the asymptotics of the radial part of the single-nucleon wave functions which depends on the particle separation energy through

$$
R(r) \propto \exp \left(-\sqrt{\frac{2 m\left|E-E_{\pi}\right|}{\hbar^{2}}} r\right)
$$

where $E$ and $E_{\pi}$ are the energies of the initial state of the $A$-nucleus and of the final state in the parent $(A-1)$-nucleus, respectively, and $m$ is the reduced nucleon mass [18].

In case the number of the parent states to be taken into account in $(A-1)$-nucleus is too large, one is obliged to introduce different approximations. There exist two basic possibilities to deal with the problem: either to truncate the number of states, leaving only those which mostly contribute to the matrix element (8), and then scale the result, or to neglect completely the dependence on $\pi$ and approximate all integrals by those corresponding to the $(A-1)$ nucleus being in its ground state. We have used the latter procedure. In this case, the expression (8) reduces to

$$
M_{ \pm}=\sum_{j_{1}, j_{2}} \operatorname{OBTD}\left(j_{1}, j_{2} ; \Delta J=1\right)\left\langle n_{2} l_{2} j_{2}\left\|\hat{\sigma} \hat{\tau}_{ \pm}\right\| n_{1} l_{1} j_{1}\right\rangle
$$

where $\operatorname{OBTD}\left(j_{1}, j_{2} ; \Delta J=1\right)$ are one-body transition densities [25]. 
We will present the results on $B(\mathrm{GT})$-values obtained using both Eq. (12) and Eq. (8), where we have taken into account up to as many as 100 states of a parent nucleus. Provided this number is still too small for a good approximation (as it is for some $s d$-shell nuclei for which the dimensions of the eigenvalue problem reaches a few thousands), we have restricted ourselves only to the calculations according to Eq. (12).

To summarize, within the shell model the asymmetry in the decay of mirror nuclei can have two origins: i) inequivalent $S^{1 / 2}$ values in (8) or OBTD's in (12) for $\beta_{+}$and $\beta_{-}$decays due to isospin breaking effects; ii) difference of the overlaps between the proton and neutron radial wave functions (10) for mirror transitions. (These two nuclear structure effects were referred to, in the past, as effects due to Coulomb and charge-dependent nuclear forces and binding energy phenomena, respectively [20].) The two factors can be estimated independently and therefore we will discuss them separately.

\section{A. Isospin corrections to configuration mixing}

The isospin symmetry in nuclei is only an approximate symmetry due to the presence of the Coulomb interaction and isospin non-conserving nuclear forces. Within the shell model, both effects can be well incorporated in a phenomenological way. In Ref. 24], the INC Hamiltonians have been derived by supplementing several empirical shell model interactions (e.g., Cohen-Kurath for $p$-shell nuclei, the interaction of Zuker and co-workers [27] within the $\left(p_{1 / 2} d_{5 / 2} s_{1 / 2}\right)$ shell model space for nuclei around ${ }^{16} \mathrm{O}$, the interaction of Wildenthal [23] for $s d$-shell nuclei) by extra terms including isospin non-conserving ones. These are isovector single-particle energies for a given shell, matrix elements of Coulomb force and of the isovector and isotensor parts of nucleon INC interaction, whose strengths were fitted to the parameters of the isobaric mass-multiplet equation. The interaction yields the shifts of the levels in mirror partners often within $100 \mathrm{keV}$ precision compared to experimental ones. We adopt these INC interactions for all our calculations within the $p,\left(p_{1 / 2} d_{5 / 2} s_{1 / 2}\right)$ and $s d$ configuration spaces.

\section{B. Radial wave functions}

We determine the single-particle radial wave function $R_{n l j}(r)$ of a nucleon participating in the $\beta$ decay by solving the Schrödinger equation with a WS potential :

$$
V(r)=-V_{w s} f(r)-V_{s o} \frac{r_{0}^{2}}{r} \frac{d}{d r}[f(r)] \mathbf{l} \cdot \mathbf{s}+V_{c} h(r)
$$

where

$$
\begin{aligned}
f(r) & =\frac{1}{\left[1+\exp \left(\frac{r-R_{0}}{a}\right)\right]} \\
h(r) & =\left\{\begin{array}{l}
\frac{1}{r} \text { for } r \geq R_{0} \\
\frac{1}{2 R_{0}}\left(3-\frac{r^{2}}{R_{0}^{2}}\right) \quad \text { for } \quad r<R_{0}
\end{array}\right.
\end{aligned}
$$

with $R_{0}=r_{0}(A-1)^{1 / 3}$ and $V_{c}=Z e^{2}$. The parameters are chosen following the Bohr-Mottelson parametrization [28], namely $r_{0}=1.27 \mathrm{fm}, a=0.75 \mathrm{fm}, V_{w s}=V_{0}-V_{N Z} *(N-Z) t_{z} / A, V_{s o}=V_{l s} * V_{w s}, V_{0}=50.5 \mathrm{MeV}, V_{N Z}=32$ $\mathrm{MeV}, V_{l s}=0.22$. $N, Z, A$ are the neutron, proton and mass numbers of the nucleus. We have checked that this parametrization reproduces well charge radii. The agreement for $p$-shell nuclei is within $5 \%$, except for the halo nucleus ${ }^{8} \mathrm{~B}$. The WS potential used in Ref. [18] does not contain any symmetry term, while the one of Ref. [20] differs in the parametrization and includes an additional Coulomb term.

Remark that the potentials we use for the mirror partners differ only for the Coulomb term.

In general, there are two possible procedures to determine the radial part of the wave functions by adjusting the energies of the single-particle states in the potential of the parent $(A-1)$-nucleus to the experimental proton or neutron separation energy in the $A$-nucleus. Method I : we fit the last unoccupied single-particle state and keep the same potential to get all the other radial wave functions. This choice was followed in Refs. 19, 20]. Method II : we adjust each single-particle energy at the separation energy of the nucleus, as was done in Ref. [18] for the $A=12$ case only. We believe that the latter procedure is more consistent with the shell model than the former because method I corresponds to the extreme independent particle model. This conclusion is also supported by the unrealistic values for the electromagnetic moments in $A=8,9$ obtained with method I. 
In practice, the adjustment of the energies is performed either by including a surface term $\left(\sim\left[f^{\prime}(r)\right]^{2}\right)$ or by varying the depth of the volume term in the potential. We use the same set of parameters fitted for a given nucleus for its mirror partner. This automatically yields single-particle energies very close to the experimental separation energies (the difference does not exceed a few hundred $\mathrm{keV}$ and in some cases $1 \mathrm{MeV}$ ).

We have studied the sensitivity of the asymmetries to both methods I and II (either including the surface term or by varying the depth of the volume term) for $p$-shell nuclei as trial case, and we have applied method II only, the most consistent one, to calculate the asymmetries for the $s d$-shell nuclei.

\section{RESULTS}

In this section we present the calculated magnetic and quadrupole moments, the transition rates and the asymmetries for an ensemble of $p$-shell and $s d$-shell nuclei. The $S^{1 / 2}$ values and OBTD's have been obtained from the shell model code Oxbash [29]. We use different types of radial wave functions : Harmonic Oscillator (HO) and Woods-Saxon (WS) wave functions obtained from the different fitting procedures as explained in the previous section (method I and II), and either neglecting the dependence on the parent state (12) or including it (8] 10). We also discuss how the results vary when one adjusts the single-particle energies either by including a surface (S) or by changing the volume $(\mathrm{V})$ term of the WS potential.

The experimental $B(\mathrm{GT})$-values are obtained following the procedure outlined in Ref. [30, 31] with updated data on $Q$-values, half-lives and branching ratios as specified in Tables II and III (from the compilation of asymmetries of allowed transitions $\left(\log (f t)<6, \pi_{i}=\pi_{f}\right)$ in $A<40$ nuclei presented in Ref. 15]). For the decay to unbound states in the $\mathrm{A}=8,9,17$ systems, we use the approximation of widthless levels and therefore we do not adopt the f-factors of Ref. [31].

For comparison with theory, we have selected only the transitions for which the error on the asymmetry is small enough, since our final purpose is to extract the contribution of the second-class currents, if any.

Before discussing the results on $B(\mathrm{GT})$-values, we comment on the problem of quenching of the GT strength. We also show what level of accuracy of the nuclear wave functions we have, by considering the electromagnetic properties of some of the nuclear states involved in the studied $\beta$-decay branches.

\section{A. Effective GT-operator}

It is was suggested long ago that the axial-vector coupling constant $g_{A}$ in GT transitions is quenched in the nuclear medium compared to the free space value (for review see, e.g., Ref. [40] and references therein). In fact, the experimentally measured total GT strength from charge-exchange reactions is largely suppressed compared to the model-independent sum rule [40], as well as the $\beta$-decay transition rates are systematically overestimated by the $0 \hbar \omega$ shell-model calculations (e.g., Ref. [30]). The reasons for this discrepancy are (i) lack of high-order configuration mixing, and (ii) inadequacy of the one-body GT operator and need of taking into account meson-exchange currents, in particular, $\Delta$-isobar admixtures (the former effect is estimated to provide a dominant contribution to the quenching within the $0 \hbar \omega$ shell model [41, 42]).

However, it is also well established [30, 43] that in the shell model, up to a good approximation, the phenomenon can be taken into account by an empirical multiplicative factor, $\left(g_{A} / g_{V}\right)_{\text {eff }}=\lambda\left(g_{A} / g_{V}\right)_{\text {bare }}$ with a bare value for the ratio of 1.26 as obtained from neutron decay (see Ref. [4] for certain cautions). In this case, the quenching does not influence asymmetries of the transition rates, i.e. the ratios of the matrix elements, which is then simply given by (77).

Note, that here we are concerned with individual transitions, which represent only a small fraction of the total GT strength, even being the main branches of the $\beta$ decay. Their analysis is more challenging because of the extreme sensitivity to configuration mixing predictions, in particular, for weak transitions or in the presence of close-lying final states. Thus, the quenching phenomenon as described above can be more clearly seen for stronger transitions.

Bearing these remarks in mind, in the sections below we give the theoretical values for the GT-transition rates obtained with $\left(g_{A} / g_{V}\right)_{\text {bare }}=1.26$.

\section{B. Test of the wave functions}

The accuracy of the wave functions is one of the most critical issues of the study, especially since we are interested in the fine details of the weak interaction. To check the wave functions of the states involved in the $\beta$ decay considered below and to demonstrate the present level of accuracy, we have computed the transition probabilities of electromagnetic processes for some of the states of interest. The electromagnetic processes are described up to an uncertainty 
arising mainly from two factors, provided the best wave functions of the many-body problem are available: i) the restricted model space used in the calculations; and ii) impulse approximations for the operators.

We have calculated some electromagnetic characteristics, namely, magnetic dipole and electric quadrupole moments for the ground states, which provide the most stringent test for the wave function of a given state. We will follow the conventional way and use the standard form of the M1 operator with the free-nucleon $g$-factors $\left(g_{s}(\pi)=5.586\right.$, $\left.g_{l}(\pi)=1.0, g_{s}(\nu)=-3.826, g_{l}(\nu)=0.0\right)$, which gives in general a reasonable agreement with the data. The electric quadrupole moments have been calculated with three different choices of effective charges [22, 45]. The results are summarized in Table I and Fig. 2(a,b), where the calculated values for certain states in $p$-shell nuclei exploited below in the $\beta$-decay analysis are compared with the data.

Both types of moments were computed according to

$$
M(L)=\sum_{j_{1}, j_{2}} \operatorname{OBTD}\left(j_{1}, j_{2} ; \Delta J=L\right)\left\langle n_{2} l_{2} j_{2}\left\|\hat{O}_{L}\right\| n_{1} l_{1} j_{1}\right\rangle,
$$

where $\hat{O}_{L}$ is magnetic dipole or electric quadrupole operator. The OBTD values have been obtained from INC interaction, while the radial wave functions from either the harmonic oscillator or the WS potential, adjusted to give the separation energies only with respect to the ground state.

The magnetic dipole moments are sensitive to the single-particle configurations and almost do not depend on the radial form of the basis wave functions. As seen from Table I and Fig. 2, the theoretical description of the magnetic moments is always within $20 \%$, thus showing the high quality of the effective interaction we use here.

The quadrupole moments depend on both the configurations involved and the radial wave functions. The results obtained with both harmonic oscillator and WS radial wave functions (method II, V) for three choices of the effective electric charges are given in Table I. The calculations obtained by fitting the surface term (S) gives very similar results and to avoid repetition we do not present them.

To get the radial wave functions, we have also tested the other possibility of adjusting the potential, namely, fitting the separation energies to the last unoccupied nucleon state (method I, V or S). We have found out that this procedure yields unrealistic values of the quadrupole moments for light nuclei. For example, the moments of ${ }^{8} \mathrm{Li}$ calculated with the radial wave functions I V and I S are -0.85 e.fm ${ }^{2}$ and $-0.88{\mathrm{e} . \mathrm{fm}^{2}}^{2}$ respectively, to be compared with the experimental values and theoretical results obtained with method II (Table I). We will see that the calculation of $\beta$-decay transition rates does not help in choosing between method I and II, because they both yield similar agreement with the measured $B(\mathrm{GT})$-values. We emphasize that only method I (V or S) was used in the past [19, 20], the case $A=12$ being the only exception [18].

As seen from Table I, both harmonic oscillator and WS radial wave functions (method II) yield similar values of the quadrupole moments and on average reproduce fairly well the experimental data. It is difficult to use the results as an additional constraint for the potential, since the quadrupole moments are also rather sensitive to the choice of the effective charges (see Table I). The values obtained with the WS wave functions and the last set of the effective charges $(e(\pi)=1.35, e(\nu)=0.35)$ are plotted in Fig. 2(b) and as seen agree within $20 \%$ with the results of the measurements.

\section{GT-transition rates}

The calculated $B(\mathrm{GT})$-values,

$$
B\left(\mathrm{GT}_{ \pm}\right)=\frac{\left(g_{A} / g_{V}\right)^{2}}{2 J_{i}+1}\left|M_{ \pm}\right|^{2}
$$

are summarized in Tables II, for $p$-shell nuclei, and III, for $s d$-shell nuclei, in comparison with the experimental data (Fig. 3). The theoretical results are organized into columns labelled by the type of calculations. Contrary to the moments discussed above, the $\beta$-decay rates are extremely sensitive to both, the asymptotics of the radial wave functions and to the correct accounting for the dependence on the parent state. First three columns with the results on $B(\mathrm{GT})$ values in both tables are obtained using formula (12), while in the last column (denoted by a star) the radial dependence on the parent state according to (8) is taken into account. The label IC(INC) refers to the isospinconserving (isospin-nonconserving) interaction used, and HO or WS indicate the type of the wave functions. Roman numbers I or II in Table II stand for the method to fit the single-particle potentials as discussed in section II. Only method II is used in the calculations for $s d$-shell nuclei. 
1. $\quad$-shell nuclei

$A=8,9$.

The analysis of light nuclei is often complicated from both theoretical and experimental sides. The studied $A=8$ triad includes the transition to the very broad $2^{+}$state of ${ }^{8} \mathrm{Be}$, unstable with respect to $\alpha \alpha$ breakup. Moreover, in the description of the well-known proton-halo nucleus ${ }^{8} \mathrm{~B}$, the vicinity of the continuum can be important. The decay of proton-rich ${ }^{9} \mathrm{C}$ always continues through $2 \alpha$-p three-body break-up, which complicates the experimental analysis of corresponding transitions 34]. In addition, the specific feature of ${ }^{9} \mathrm{Li}^{-}{ }^{9} \mathrm{C}$ mirror pair is the so-called anomalous isoscalar magnetic moment [48] which is still not fully understood from a theoretical point of view.

The shell model yields good agreement of the energy spectra and even nuclear moments with either harmonic oscillator or WS wave functions (see Table I) for the lowest states of $A=8,9$, despite missing the width of the unbound states. The only significant deviation is the quadrupole moment of the ground state of ${ }^{8} \mathrm{~B}$, showing necessity to go beyond the standard shell model we use.

However, as is clearly seen from Table II, the shell model experiences difficulties in reproducing the absolute values of the GT $\beta$-decay rates which are about ten and five times overestimated for $A=8$ and $A=9$ systems, respectively. The calculations with the best effective GT operator of Ref. 31] also overestimates the transition rates in $A=8,9$, although with a factor of two discrepancy.

The apparent disagreement is unlikely to be the consequence of a particular renormalization of the GT operator for these nuclei, and is an indication of the limits of the theoretical approach used (few-nucleon systems, clusterization effects, close continuum). Following these arguments, one should not consider data on $A=8,9$ to extract secondclass currents, until an accurate treatment by more fundamental microscopic techniques, such as Green's Function Monte-Carlo methods [52], and no-core shell model [53] is attained. Note, that $A=8$ nuclei treated by the standard shell model have mainly been used in the past in the context of the second-class currents problem (e.g., [5, 19, 20]).

$A=12$.

The triad of $A=12$ nuclei, ${ }^{12} \mathrm{~B}\left(1^{+} ; 1\right) \rightarrow{ }^{12} \mathrm{C}\left(0^{+} ; 0\right)$ and ${ }^{12} \mathrm{~N}\left(1^{+} ; 1\right) \rightarrow{ }^{12} \mathrm{C}\left(0^{+} ; 0\right)$, is the most famous and well studied case [54], since it was the first measured case of large asymmetry and up to now with the best precision. The electromagnetic moments for $1^{+}, T=1$ states in mother nuclei are well reproduced. The rates of the GT transitions to the ground state of ${ }^{12} \mathrm{C}$ are close to the measured ones, while the calculated $\beta$-decay rates to the first excited state are about two times larger than the experimental values. The use of effective GT-operator [31] helps to reduce the disagreement to only $20 \%$ of overestimation.

At present, there are already $a b$-initio shell-model calculations available for ${ }^{12} \mathrm{C}$ [55. It would be interesting to apply the model to calculate the GT strength, since the questions such as limited configuration space and necessity of auxiliary realistic potential are not encountered there.

$A=13$.

The data on the rates for the GT transitions to the ground states $\left(\frac{1}{2}^{-}, \frac{1}{2}^{-}\right)$in $A=13$ nuclei is also advantageous by its precision. The shell model well reproduces the four lowest excited states of negative parity, including the values of the ground state magnetic and quadrupole moments. The positive parity states are outside of the $p$-shell model space. The theoretical $\beta$-decay rates are a factor of 1.2 larger than the experimental ones. No particular differences between wave functions obtained with the fitting procedure I and II can be seen in this case.

\section{2. sd-shell nuclei}

\section{$A=1 \%$.}

We will devote a special discussion to $A=17$ nuclei, since they have already got a lot of attention from both experimental and theoretical sides, because of the enormously large asymmetries of the first forbidden $\beta$-decay transition rates [56, 57, 58].

Here we study these nuclei with respect to the asymmetries in the allowed GT decay modes, which are also large. Although the nuclei near the doubly magic ones are the most difficult for the shell model framework, nuclei around ${ }^{16} \mathrm{O}$ can be well described within $p_{1 / 2} d_{5 / 2} s_{1 / 2}$-shell model space [27]. In the calculations we used the interaction of Ref. [24], which is an INC version of the interaction close to the pioneer one [27]. Let us remark that the interaction exploited here gives much more realistic spectra, in particular the positions of $3 / 2^{-}$states in ${ }^{17} \mathrm{O}$ and ${ }^{17} \mathrm{~F}$, and transition rates as compared to the $(p s d)$-shell model interaction used in Ref. [31].

$A=20,25,28,31,35$.

As is well-known, the $s d$-shell nuclei are fairly good described by the interaction of Wildenthal [22, 23], while the INC corrections take care about the subtle effects. The systematic calculations of $\beta$-decay rates in $s d$-shell nuclei have been already performed [22, 30], although without accounting for INC effects, and are known to be in a good agreement with the experiment. 
The results on $B(\mathrm{GT})$-values for $s d$-shell nuclei, obtained with both IC and INC Hamiltonians and with harmonic oscillator or WS radial wave functions are summarized in Table III. There are still no data on the transitions for $A=21$ and $A=25$ nuclei, however, the measurements are under way [59]. The calculated $B(\mathrm{GT})$ values are all about a factor of $0.5-0.7$ quenched compared to the experiment, except for the $A=20$ case ( $T=2 \rightarrow T=1$ transitions).

As in the case of $p$-shell nuclei, there was no principal difference between the radial wave functions, however, the values obtained with formula (8) are assumed to be the most realistic ones. We did not take into account the dependence on the parent states for $A=25,28,31$ because of the extremely large dimensions of the model spaces, as well as for $A=35$ (S-CL-K-Ar) for which the experimental error bars are very large.

\section{Asymmetries of GT transition rates}

We have performed, for the first time, a detailed study of the asymmetries for a selected ensemble of $p$ - and $s d$ shell nuclei. The chosen nuclei are on one hand rather well described by the shell model and on the other hand the corresponding measured asymmetries present small error bars (Table IV and Fig. 4).

We have calculated the asymmetries of the $B(\mathrm{GT})$-values in the mirror nuclei due either to the use of the isospin non-conserving (INC) interaction or/and to differences in the tails of wave functions of the decaying proton or neutron. We present the effect of the isospin non-conserving interaction alone (INC+HO), of the mismatch of the radial integrals alone $(\mathrm{IC}+\mathrm{WS})$ and of both $(\mathrm{INC}+\mathrm{WS})$. In the latter case, we show the influence of the inclusion of the dependence on the parent states in the radial wave functions $\left((\mathrm{INC}+\mathrm{WS})^{*}\right)$.

We also present the first estimates of the asymmetries for some $s d$-shell nuclei for which the shell model description is rather good, but the asymmetries either present too large error bars or their analysis is under study [15] (Table V and Fig. 4).

All the results shown in Tables IV and V correspond to the radial wave functions obtained with method II by varying the volume term of the potential in the fitting procedure. We have checked that fitting the single-particle energies with the inclusion of a surface term changes the results by a few percent. We have also calculated the asymmetries using method I. This always leads to much larger asymmetries than those determined by method II and, in particular, it gives unrealistic ones for cases where one single-particle state is unbound, such as for $A=8,9$. We have also mentioned above that it leads to unrealistic results for the quadrupole moments of some light nuclei. For these reasons and because of a better consistency with the shell model, we believe that method II (and not method I) should be used in the procedure of fitting the wave functions contrary to the previous studies [19, 20].

As we can see from Table IV, the effect due to the differences in the tails of the wave functions is by far the largest in the $p$-shell nuclei; whereas in the case of the $s d$-shell nuclei, it can be sometimes of the same order as the asymmetry due to the INC interaction. This happens when there is a large contribution of the $d_{5 / 2}$ or $d_{3 / 2}$ single-particle states for which the centrifugal barrier tends to confine the wave function inside the potential reducing the differences in the tails. The case $A=17$, including the halo nucleus ${ }^{17} \mathrm{~F}$, represents an exception : the asymmetry due to the INC interaction is almost ten times larger than the one due to the mismatch of the wave functions.

Concerning the dependence on the parent states (Eq. (8) ), we see from Table IV that its inclusion has a significant effect on the asymmetries. As it was found in [19, 20], the use of Eq.(8) instead of Eq.(10) may significantly increase or decrease the asymmetry and in a few cases up to a factor of two.

We have compared the theoretical asymmetries to the ones obtained in Ref. 19, 20], although those calculations differ from ours in the parametrization of the shell model Hamiltonian, the WS potential, as well as in the fitting procedure (method I was used). In general, our asymmetries are much larger than previously calculated in Ref. 19, 20]. However, for $A=12$ case, our result is in reasonable agreement with their values, as well as with the value in [18] where method II was used and also a very similar parametrization of the WS potential.

As far as the comparison with the measured asymmetries is concerned (Table IV), we see that our calculated asymmetries due to the only approximate isospin symmetry in the nucleus (described in our case through the use of the INC interaction 24] and of WS radial wave functions) : a) are compatible with the measured asymmetries in a few cases; b) overestimate the measured values for $A=8,12($ g.s. $), 17$; c) underestimate the experiments for $A=9,20$ (Fig. 4). Remark that $A=8,17$ represent rather delicate cases because they include halo nuclei which should be treated with particular techniques. For $A=8,9$, the theoretical $B(\mathrm{GT})$-values are larger than the experimental ones by a factor of four.

For $p$-shell nuclei, these conclusions do not change even after the inclusion of the other above mentioned higher order corrections to $\delta^{\text {nucl }}$, which have been found in Ref. [20] not to exceed $1 \%$. 


\section{CONCLUSIONS}

Within the shell model, we have calculated the Gamow-Teller $\beta_{+}$and $\beta_{-}$decay rates for an ensemble of light $p$ - and $s d$-shell mirror nuclei. The theoretical results are important in connection with the possible existence of second-class currents in the weak interaction. To assess their existence, it is necessary to carry out accurate estimates of the asymmetries coming from nuclear structure aspects.

The calculations are performed systematically for the known experimental cases among $p$-shell and, for the first time, $s d$-shell nuclei. We have evaluated the asymmetry due to the only approximate isospin symmetry of the shell model Hamiltonian and differences between the proton and neutron radial wave functions. The former is accounted for through the use of an isospin non-conserving interaction which describes well the shifts in energy levels of the mirror nuclei. The latter is due to the use of realistic single-particle radial wave functions from a WS potential adjusted to reproduce the experimental proton and neutron separation energies.

We have thoroughly studied the sensitivity of the asymmetries to the inclusion of the parent state dependence as well as to different fitting procedures, namely by fitting either the energy of the last unoccupied state or the energies of all the single-particle states in the shell at the same experimental separation energy. We argue that, contrary to what was done in the past, the latter fitting procedure is more consistent than the former.

The theoretical asymmetries we obtain are on average larger than all previous estimates. The results are compatible with the measured values in a few cases. For $A=8,12,17$, the theoretical values are larger than the experimental ones, whereas for $A=9,20$ we find the opposite.

As is clear from the present study, the average reliability of $B(\mathrm{GT})$-values, which determines the level of confidence for the theoretical asymmetries, seems still not to be high enough for extracting the second-class currents from the differences in nuclear GT $\beta$-decay rates, even at the present level of many-body techniques.

In this work, we restricted ourselves to WS wave functions. Calculations with Hartree-Fock wave functions would not modify the quality of the $B(\mathrm{GT})$-values significantly enough to change these conclusions. Nevertheless, these calculations would be needed, once a better overall description of the transition rates is attained.

More extensive and systematic data would be very helpful.

\section{Acknowledgments}

We thank Bertram Blank and Jean-Charles Thomas for making us aware of the problem, providing us with the systematics of available experimental data prior to publication and stimulating interest to our work at earlier stages. We are grateful to Nicole Vinh Mau for her kind help at the beginning of our work and the very interesting numerous discussions. We gratefully acknowledge the discussions, also in e-mail correspondence, with H. Sazdjian and E. Ormand. N.A.S. thanks IPN Orsay for hospitality and CNRS, France, for visitor support, and acknowledges the Institute for Nuclear Theory at the University of Washington for hospitality and the U.S. Department of Energy for partial support during the completion of this work. N.A.S. thanks K. Heyde for valuable comments. Financial support of the DWTC (grant IUAP \#P5/07) is acknowledged.

[1] S. Weinberg, Phys. Rev. 112 (1958) 1375.

[2] J. N. Huffaker and E. Greuling, Phys. Rev. 132 (1963) 738.

[3] J. Delorme and M. Rho, Nucl. Phys. B34 (1971) 317; K. Kubodera, J. Delorme and M. Rho, Nucl. Phys. B66 (1973) 253; Phys. Rev. Lett. 38 (1977) 321.

[4] L. Grenacs, Ann. Rev. Nucl. Part. Sci. 35 (1985) 455.

[5] D. H. Wilkinson, Eur. Phys. J. A 7 (2000) 7.

[6] N.C. Mukhopadhyay, Proceedings to the "WEIN-98" conference, 1998, Santa Fe and nucl-th/9810039

[7] J. Bartlet et al, Phys. Rev. Lett. 76 (1996) 4119.

[8] R. Balest et al, Phys. Rev. Lett. 75 (1995) 3809; D.Buskalic et al, Z. Phys. C 74 (1997) 263.

[9] B. Holstein, Phys. Rev. C 29, 623 (1984); M. Morita, Hyp. Int. 21, 143 (1985).

[10] J. Govaerts, hep-ph/9701385

[11] T. Minamisono et al., Phys. Rev. Lett. 80, 4132 (1998).

[12] L. De Braeckeleer, Phys. Rev. C 45 (1992) 1935; L. De Braeckeleer et al, Phys. Rev. C 51 (1995) 2778.

[13] J. F. Amsbaugh, M. Beck, L. De Braeckeleer et al, Proc. 6th Conf. "Intersections between Particle and Nuclear Physics" Ed. T.W.Donnelly (AIP, 1997); p.416. 
[14] D. H. Wilkinson, Phys. Lett. B 31 (1970) 447.

[15] J.C. Thomas, Ph.D. thesis (University of Bordeaux, 2002); ftp://ftpcenbg.in2p3.fr/pub/nex/thomas/

[16] H. J. Lipkin, Phys. Lett. B 34 (1971) 202.

[17] R. J. Blin-Stoyle and M. Rosina, Nucl. Phys. 70 (1965) 321.

[18] J. Blomqvist, Phys. Lett. B 35 (1971) 375.

[19] D. H. Wilkinson, Phys. Rev. Lett. 27 (1971) 1018.

[20] I. S. Towner, Nucl. Phys. A 216 (1973) 589.

[21] S. Cohen and D. Kurath, Nucl. Phys. 73 (1965) 1.

[22] B. A. Brown and B. H. Wildenthal, Ann. Rev. Nucl. Part. Sci. 38 (1988) 29; B. A. Brown, Progr. Part. Nucl. Phys. 47 (2001) 517.

[23] B. H. Wildenthal, Progr. Part. Nucl. Phys. 11 (1984) 5.

[24] W. E. Ormand and B. A. Brown, Nucl. Phys. A 491 (1989) 1.

[25] W. E. Ormand and B. A. Brown, Nucl. Phys. A 440 (1985) 274.

[26] P. J. Brussaard and P. W. M. Glaudemans, Shell-Model Applications in Nuclear Spectroscopy (Amsterdam: North-Holland Publishing, 1977)

[27] A. P. Zuker, B. Buck and J. B. McGrory, Phys. Rev. Lett. 21 (1968) 39; A. P. Zuker, Phys. Rev. Lett. 23 (1969) 983.

[28] A. Bohr, B. R. Mottelson, Nuclear Structure (Benjamin, Menlo Park, 1969), Vol 1.

[29] B. A. Brown, A. Etchegoyen and W. D. M. Rae, The computer code Oxbash, MSU-NSCL Report No. 524 (1984).

[30] B. A. Brown and B. H. Wildenthal, At. Data Nucl. Data Tables 33 (1985) 347.

[31] W.-T. Chou, E. K. Warburton, B. A. Brown, Phys. Rev. C 47 (1993) 163.

[32] G. Audi et al, Nucl. Phys. A 624 (1997) 1.

[33] G. Nyman et al, Nucl. Phys. A 510 (1990) 189.

[34] F. Gete et al, Phys. Rev. C 61 (2000) 064310; U. C. Bergmann et al, Nucl. Phys. A 692 (2001) 427.

[35] D. Tilley et al, Nucl. Phys. A 564 (1993) 1; D. Tilley et al, Nucl. Phys. A 636 (1998) 249.

[36] M. Domsky et al, Phys. Rev. C 49 (1994) 1867.

[37] P.M. Endt, Nucl. Phys. A 521 (1990) 1; P.M. Endt, Nucl. Phys. A 633 (1998) 1.

[38] H.O.U. Fynbo et al, Nucl. Phys. A 677 (2000) 38.

[39] W. Trinder et al, Phys. Lett. B 459 (1999) 67.

[40] F. Osterfeld, Rev. Mod. Rhys. 64 (1992) 491.

[41] B. A. Brown and B. H. Wildenthal, Nucl. Phys. A 474 (1987) 290.

[42] I. S. Towner, Phys. Rep. 155 (1987) 264; A. Arima et al, Adv. Nucl. Phys. 18 (1987) 1.

[43] D. H. Wilkinson, Phys. Rev. C 7 (1973) 930; Nucl. Phys. A 209 (1973) 470; Nucl. Phys. A 225 (1974) 365.

[44] B. A. Brown, Phys. Rev. Lett. 69 (1992) 1034.

[45] B. A. Brown, B. H. Wildenthal, W. Chung, et al, Phys. Rev. C 26 (1982) 2247.

[46] P. Raghavan, At. Data Nucl. Data Tables, 42 (1989) 194.

[47] T. Minamisono et al, Phys. Rev. Lett. 69 (1992) 2058.

[48] K. Matsuta et al, Nucl. Phys. A 588 (1995) 153c; M. Huhta, et al, Phys. Rev. C 57 (1998) R2790.

[49] T. Minamisono et al, Phys. Lett. B 420 (1998) 31.

[50] K. Matsuta et al, Hyperfine Interact. 97/98 (1996) 519.

[51] K. Matsuta et al, Phys. Lett. B 459 (1999) 81.

[52] R. B. Wiringa, S. C. Pieper, J. Carlson, V. R. Pandharipande Phys. Rev. C 62 (2000) 014001.

[53] P. Navratil, B. R. Barrett, Phys. Rev. C57 (1998) 3119.

[54] R. W. Peterson and N. W. Glass, Phys. Rev. 130 (1963) 292; T. R. Fisher, Phys. Rev. 130 (1963) 2388.

[55] P. Navratil, J. P. Vary, B. R. Barrett, Phys. Rev. Lett. 84 (2000) 5728.

[56] M. J. G. Borge et al, Phys. Lett. B 317 (1993) 25.

[57] D. J. Millener, Phys. Rev. C 55 (1997) R1633.

[58] N. Michel, J.Okołowicz, F. Nowacki, M. Płoszajczak, Nucl. Phys. A 703 (2002) 202.

[59] J.C. Thomas, B. Blank, private communication. 
TABLE I: Comparison of experimental and theoretical static magnetic dipole $(\mu)$ in $\mu_{N}$ and electric quadrupole $(Q)$ in e.fm ${ }^{2}$ moments of the ground states of the $p$-shell nuclei of interest. The quadrupole moments have been calculated either with harmonic oscillator (HO) or Woods-Saxon (WS) wave functions using method II with a volume term (see text), according to [15]. The experimental data are taken from Ref. [46], if not specified.

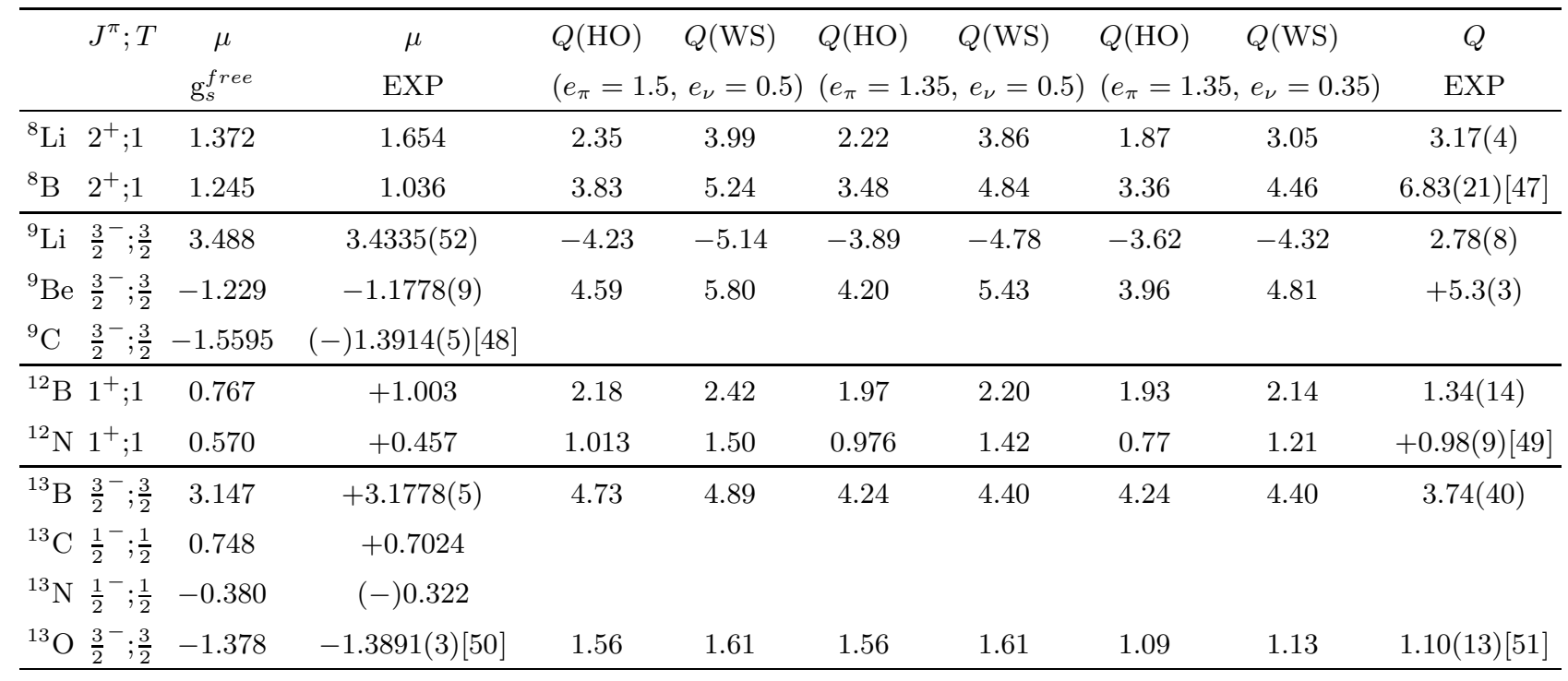

TABLE II: Comparison of experimental and theoretical $B(\mathrm{GT})$-values in $p$-shell mirror nuclei. The results shown are obtained with the isospin-nonconserving (INC) interaction and either harmonic oscillator (HO) or Woods-Saxon (WS) wave functions determined with method I or II (see text) by adjusting the volume term, without including the dependence on the parent state. The results obtained by including a surface term are shown into parenthesis. The $B(\mathrm{GT})$-values given in column (INC+WS)* incorporate the dependence on the parent states as follows from Eq. [8). The experimental values 15] are obtained following the procedure of Refs. [30, 31] on the basis of $Q$-values from Ref. [32], halflives and branching ratios from Ref. [31], if not specified.

\begin{tabular}{|c|c|c|c|c|c|c|c|}
\hline & $J_{i}^{\pi} ; T_{i}$ & $J_{f}^{\pi} ; T_{f}$ & $\mathrm{INC}+\mathrm{HO}$ & I: INC+WS & II: INC+WS & II: $(\mathrm{INC}+\mathrm{WS})^{*}$ & EXP \\
\hline${ }^{8} \mathrm{Li}\left(\beta^{-}\right){ }^{8} \mathrm{Be}$ & $2^{+} ; 1$ & $2_{1}^{+} ; 0$ & 0.1170 & $0.0265(0.0295)$ & $0.1006(0.1001)$ & 0.0984 & $0.0158(2)$ \\
\hline${ }^{8} \mathrm{~B}\left(\beta^{+}\right)^{8} \mathrm{Be}$ & $2^{+} ; 1$ & $2_{1}^{+} ; 0$ & 0.1135 & $0.0082(0.0092)$ & $0.0863(0.0864)$ & 0.0828 & $0.0146(2)$ \\
\hline${ }^{9} \mathrm{Li}\left(\beta^{-}\right){ }^{9} \mathrm{Be}$ & $\frac{3}{2}^{-} ; \frac{3}{2}$ & $\frac{3}{2}{ }_{1}^{-} ; \frac{3}{2}$ (g.s.) & 0.1498 & $0.1052(0.1101)$ & $0.1375(0.1352)$ & 0.1417 & $0.0296(18)$ [33] \\
\hline${ }^{9} \mathrm{C}\left(\beta^{+}\right){ }^{9} \mathrm{~B}$ & $\frac{3}{2}^{-} ; \frac{3}{2}$ & $\frac{3}{2}{ }_{1}^{-} ; \frac{3}{2}$ (g.s.) & 0.1517 & $0.0740(0.0778)$ & $0.1310(0.1283)$ & 0.1379 & $0.0256(3)[34]$ \\
\hline \multirow[t]{2}{*}{${ }^{12} \mathrm{~B}\left(\beta^{-}\right)^{12} \mathrm{C}$} & $1^{+} ; 1$ & $0_{1}^{+} ; 0$ (g.s.) & 0.5078 & $0.4250(0.4528)$ & $0.4703(0.4692)$ & 0.4250 & $0.5272(18)$ \\
\hline & & $2_{1}^{+} ; 0$ & 0.1038 & $0.0979(0.0999)$ & $0.0990(0.0984)$ & 0.0954 & $0.0476(2)$ \\
\hline \multirow[t]{2}{*}{${ }^{12} \mathrm{~N}\left(\beta^{+}\right){ }^{12} \mathrm{C}$} & $1^{+} ; 1$ & $0_{1}^{+} ; 0$ (g.s.) & 0.5045 & $0.3629(0.3918)$ & $0.4272(0.4245)$ & 0.3488 & $0.4682(31)$ \\
\hline & & $2_{1}^{+} ; 0$ & 0.1045 & $0.0875(0.0897)$ & $0.0917(0.0909)$ & 0.0857 & $0.0435(8)$ \\
\hline${ }^{13} \mathrm{~B}\left(\beta^{-}\right){ }^{13} \mathrm{C}$ & $\frac{3}{2}^{-} ; \frac{3}{2}$ & $\frac{1}{2}_{1}^{-} ; \frac{1}{2}$ (g.s.) & 0.767 & $0.6861(0.7096)$ & $0.7206(0.7218)$ & 0.7181 & $0.5671(72)$ \\
\hline${ }^{13} \mathrm{O}\left(\beta^{+}\right){ }^{13} \mathrm{~N}$ & $\frac{3}{2}^{-} ; \frac{3}{2}^{2}$ & $\frac{1}{2}_{1}^{-} ; \frac{1}{2}$ (g.s.) & 0.752 & $0.6095(0.6381)$ & $0.6580(0.6615)$ & 0.6609 & $0.5097(130)$ \\
\hline
\end{tabular}


TABLE III: Comparison of experimental and theoretical $B(\mathrm{GT})$-values in $s d$-shell mirror nuclei. We present results obtained with the isospin conserving (IC) and isospin non-conserving (INC) interactions and either harmonic oscillator (HO) or WoodsSaxon (WS) wave functions determined from method II by adjusting a volume term. The results including the dependence on the parent state are shown in column $(\mathrm{INC}+\mathrm{WS})^{*}$ only for the nuclei considered in the discussion of the asymmetries. The experimental values [15] are obtained following the procedure of Refs. [30, 31] on the basis of $Q$-values from Ref. [32], halflives and branching ratios as specified.

\begin{tabular}{|c|c|c|c|c|c|c|c|}
\hline & $J_{i}^{\pi} ; T_{i}$ & $J_{f}^{\pi} ; T_{f}$ & $\mathrm{INC}+\mathrm{HO}$ & $\mathrm{IC}+\mathrm{WS}$ & $\mathrm{INC}+\mathrm{WS}$ & $(\mathrm{INC}+\mathrm{WS})^{*}$ & EXP \\
\hline${ }^{17} \mathrm{~N}\left(\beta^{-}\right){ }^{17} \mathrm{O}$ & $\frac{1}{2}^{-} ; \frac{3}{2}$ & $\frac{3}{2}_{1}^{-} ; \frac{1}{2}$ & 0.2079 & 0.2333 & 0.2021 & 0.2023 & $0.2342(89)[35]$ \\
\hline${ }^{17} \mathrm{Ne}\left(\beta^{+}\right){ }^{17} \mathrm{~F}$ & $\frac{1}{2}^{-} ; \frac{3}{2}$ & $\frac{3}{2}_{1}^{-} ; \frac{1}{2}$ & 0.1356 & 0.2216 & 0.1272 & 0.1228 & $0.1632(50)[35,36]$ \\
\hline${ }^{20} \mathrm{~F}\left(\beta^{-}\right){ }^{20} \mathrm{Ne}$ & $2^{+} ; 1$ & $2_{1}^{+} ; 0$ & 0.0921 & 0.0948 & 0.0922 & 0.0924 & $0.0645(1)[35]$ \\
\hline${ }^{20} \mathrm{Na}\left(\beta^{+}\right){ }^{20} \mathrm{Ne}$ & $2^{+} ; 1$ & $2_{1}^{+} ; 0$ & 0.0935 & 0.1098 & 0.1085 & 0.0914 & $0.0633(4)[35]$ \\
\hline${ }^{20} \mathrm{O}\left(\beta^{-}\right){ }^{20} \mathrm{~F}$ & $0^{+} ; 2$ & $1_{1}^{+} ; 1$ & 0.9124 & 0.9571 & 0.9183 & 0.9031 & $1.1166(48)[35]$ \\
\hline${ }^{20} \mathrm{Mg}\left(\beta^{+}\right){ }^{20} \mathrm{Na}$ & $0^{+} ; 2$ & $1_{1}^{+} ; 1$ & 0.9120 & 0.9439 & 0.9054 & 0.9171 & $0.9445(666)[32,35]$ \\
\hline \multirow[t]{3}{*}{${ }^{21} \mathrm{~F}\left(\beta^{-}\right){ }^{21} \mathrm{Ne}$} & $\frac{5}{2}^{+} ; \frac{3}{2}$ & $\frac{3}{2}_{1}^{+} ; \frac{1}{2}$ (g.s.) & 0.0215 & 0.0226 & 0.0215 & & \\
\hline & & $\frac{5}{2}_{1}^{+} ; \frac{1}{2}$ & 0.1188 & 0.1243 & 0.1195 & & \\
\hline & & $\frac{7}{2}_{1}^{+} ; \frac{1}{2}$ & 0.1159 & 0.1165 & 0.1168 & & \\
\hline \multirow[t]{3}{*}{${ }^{21} \mathrm{Mg}\left(\beta^{+}\right){ }^{21} \mathrm{Na}$} & $\frac{5}{2}^{+} ; \frac{3}{2}$ & $\frac{3}{2}_{1}^{+} ; \frac{1}{2}$ (g.s.) & 0.0224 & 0.0218 & 0.0216 & & \\
\hline & & $\frac{5}{2}_{1}^{+} ; \frac{1}{2}$ & 0.1209 & 0.1214 & 0.1188 & & \\
\hline & & $\frac{7}{2}_{1}^{+} ; \frac{1}{2}$ & 0.1128 & 0.1149 & 0.1120 & & \\
\hline \multirow[t]{4}{*}{${ }^{25} \mathrm{Na}\left(\beta^{-}\right){ }^{25} \mathrm{Mg}$} & $\frac{5}{2}^{+} ; \frac{3}{2}$ & $\frac{5}{2}_{1}^{+} ; \frac{1}{2}$ (g.s.) & 0.0458 & 0.0472 & 0.0463 & & \\
\hline & & $\frac{3}{2}_{1}^{+} ; \frac{1}{2}$ & 0.0836 & 0.0820 & 0.0830 & & \\
\hline & & $\frac{7}{2}_{1}^{+} ; \frac{1}{2}$ & 0.0634 & 0.0615 & 0.0643 & & \\
\hline & & $\frac{5}{2}{ }_{2}^{+} ; \frac{1}{2}$ & 0.0048 & 0.0052 & 0.0047 & & \\
\hline \multirow[t]{4}{*}{${ }^{25} \mathrm{Si}\left(\beta^{+}\right){ }^{25} \mathrm{Al}$} & $\frac{5}{2}^{+} ; \frac{3}{2}$ & $\frac{5}{2}_{1}^{+} ; \frac{1}{2}$ (g.s.) & 0.0463 & 0.0462 & 0.0458 & & \\
\hline & & $\frac{3}{2}_{1}^{+} ; \frac{1}{2}$ & 0.0765 & 0.0798 & 0.0738 & & \\
\hline & & $\frac{7}{2}{ }_{1}^{+} ; \frac{1}{2}$ & 0.0589 & 0.0595 & 0.0578 & & \\
\hline & & $\frac{5}{2}_{2}^{+} ; \frac{1}{2}$ & 0.0049 & 0.0053 & 0.0050 & & \\
\hline${ }^{28} \mathrm{Al}\left(\beta^{-}\right){ }^{28} \mathrm{Si}$ & $3^{+} ; 1$ & $2_{1}^{+} ; 0$ & 0.1458 & 0.1444 & 0.1435 & & $0.0825(1)[37]$ \\
\hline${ }^{28} \mathrm{P}\left(\beta^{+}\right){ }^{28} \mathrm{Si}$ & $3^{+} ; 1$ & $2_{1}^{+} ; 0$ & 0.1449 & 0.1303 & 0.1287 & & $0.0870(9)[37]$ \\
\hline${ }^{31} \mathrm{Al}\left(\beta^{-}\right){ }^{31} \mathrm{Si}$ & $\frac{5}{2}^{+} ; \frac{5}{2}$ & $\frac{3}{2}_{1}^{+} ; \frac{3}{2}$ (g.s.) & 0.2120 & 0.2090 & 0.2124 & & $0.1049(122)[37]$ \\
\hline${ }^{31} \mathrm{Ar}\left(\beta^{+}\right){ }^{31} \mathrm{Cl}$ & $\frac{5}{2}^{+} ; \frac{5}{2}$ & $\frac{3}{2}{ }_{1}^{+} ; \frac{3}{2}$ (g.s.) & 0.1947 & 0.1958 & 0.1827 & & $0.0788(53)[38]$ \\
\hline${ }^{35} \mathrm{~S}\left(\beta^{-}\right){ }^{35} \mathrm{Cl}$ & $\frac{3}{2}^{+} ; \frac{3}{2}$ & $\frac{3}{2}_{1}^{+} ; \frac{1}{2}$ (g.s.) & 0.0861 & 0.0870 & 0.0865 & & $0.0588(2)[37]$ \\
\hline${ }^{35} \mathrm{~K}\left(\beta^{+}\right){ }^{35} \mathrm{Ar}$ & $\frac{3}{2}^{+} ; \frac{3}{2}$ & $\frac{3}{2}_{1}^{+} ; \frac{1}{2}$ (g.s.) & 0.0846 & 0.0843 & 0.0824 & & $0.0496(84)[37]$ \\
\hline${ }^{35} \mathrm{P}\left(\beta^{-}\right){ }^{35} \mathrm{~S}$ & $\frac{1}{2}^{+} ; \frac{5}{2}$ & $\frac{1}{2}_{1}^{+} ; \frac{3}{2}$ & 0.9052 & 0.8572 & 0.8836 & 0.8861 & $0.4591(70)[39]$ \\
\hline${ }^{35} \mathrm{Ca}\left(\beta^{+}\right){ }^{35} \mathrm{~K}$ & $\frac{1}{2}^{+} ; \frac{5}{2}$ & $\frac{1}{2}_{1}^{+} ; \frac{3}{2}$ & 0.8572 & 0.7435 & 0.7257 & 0.7458 & $0.3891(154)[39]$ \\
\hline
\end{tabular}


TABLE IV: Comparison of experimental and theoretical asymmetries $\delta(\%)$ of $\mathrm{B}(\mathrm{GT})$-values for transitions in selected $p-$ and $s d$-shell mirror nuclei. Entries denoted $A=20, A=20$ (a) and $A=35$ correspond to O-F-Mg-Na, F-Ne-Na and PS-Ca-K systems, respectively (see Table II for the details). The different columns give the asymmetries obtained with the isospin conserving (IC) or isospin non-conserving (INC) interactions and harmonic oscillator (HO) or Woods-Saxon (WS) wave functions obtained with method II (see text), adjusting a volume term. The column (INC+WS) ${ }^{*}$ shows the results obtained with the inclusion of the dependence on the parent state. The experimental asymmetries are calculated on the basis of the data from Tables II - III (see also are Ref. [15]).

\begin{tabular}{|c|c|c|c|c|c|c|c|}
\hline$A$ & $J_{i}^{\pi} ; T_{i}$ & $J_{f}^{\pi} ; T_{f}$ & $\mathrm{INC}+\mathrm{HO}$ & $\mathrm{IC}+\mathrm{WS}$ & $\mathrm{INC}+\mathrm{WS}$ & $(\mathrm{INC}+\mathrm{WS})^{*}$ & EXP. \\
\hline 8 & $2^{+} ; 1$ & $2_{1}^{+} ; 0$ & 3.10 & 13.21 & 16.65 & 18.82 & $8.4 \pm 1.8$ \\
\hline 9 & $\frac{3}{2}^{-} ; \frac{3}{2}$ & $\frac{3}{2}_{1}^{-} ; \frac{1}{2}$ (g.s.) & -1.26 & 6.4 & 4.95 & 2.72 & $16 \pm 8$ \\
\hline \multirow[t]{2}{*}{12} & $1^{+} ; 1$ & $0_{1}^{+} ; 0$ (g.s.) & 0.70 & 9.4 & 10.09 & 21.85 & $12.6 \pm 0.8$ \\
\hline & & $2_{1}^{+} ; 0$ & -0.65 & 8.45 & 7.92 & 11.33 & $9.5 \pm 1.9$ \\
\hline 13 & $\frac{3}{2}^{-} ; \frac{3}{2}^{2}$ & $\frac{1}{2}_{1}^{-} ; \frac{1}{2}$ (g.s.) & 2.10 & 7.26 & 9.50 & 8.65 & $11.3 \pm 3.2$ \\
\hline 17 & $\frac{1}{2}^{-} ; \frac{3}{2}$ & $\frac{3}{2}_{1}^{-} ; \frac{1}{2}$ & 53.26 & 5.29 & 58.90 & 64.80 & $44 \pm 7$ \\
\hline 20 & $0^{+} ; 2$ & $1_{1}^{+} ; 1$ & 0.04 & 1.41 & 1.42 & -1.53 & $18 \pm 8$ \\
\hline $20(\mathrm{a})$ & $2^{+} ; 1$ & $2_{1}^{+} ; 0$ & -1.45 & -13.64 & -14.99 & 1.11 & $1.8 \pm 0.7$ \\
\hline 35 & $\frac{1}{2}^{+} ; \frac{5}{2}$ & $\frac{1}{2}_{1}^{+} ; \frac{3}{2}$ & 5.59 & 15.28 & 21.75 & 18.81 & $18 \pm 5$ \\
\hline
\end{tabular}

TABLE V: Comparison of experimental and theoretical asymmetries $\delta(\%)$ of $\mathrm{B}(\mathrm{GT})$-values for transitions in $s d$-shell mirror nuclei. The entry denoted as $A=35$ correspond to the S-Cl-K-Ar system (see Table II for the details). The different columns give the asymmetries obtained with the isospin conserving (IC) and isospin non-conserving (INC) interactions with the harmonic oscillator (HO) or Woods-Saxon (WS) wave functions obtained with method II (see text), adjusting a volume term. The experimental asymmetries are calculated on the basis of the data from Tables II - III (see also are Ref. [15]).

\begin{tabular}{|c|c|c|c|c|c|}
\hline$A \quad J_{i}^{\pi} ; T_{i}$ & $J_{f}^{\pi} ; T_{f}$ & $\mathrm{INC}+\mathrm{HO}$ & $\mathrm{IC}+\mathrm{WS}$ & $\mathrm{INC}+\mathrm{WS}$ & EXP. \\
\hline \multirow[t]{3}{*}{$21 \frac{5}{2}^{+} ; \frac{3}{2}$} & $\frac{3}{2}_{1}^{+} ; \frac{1}{2}$ (g.s.) & -3.96 & 3.49 & -0.56 & \\
\hline & $\frac{5}{2}_{1}^{+} ; \frac{1}{2}$ & -1.71 & 2.35 & 0.60 & \\
\hline & $\frac{7}{2}_{1}^{+} ; \frac{1}{2}$ & 2.78 & 1.44 & 4.23 & \\
\hline \multirow[t]{4}{*}{$25 \frac{5}{2}^{+} ; \frac{3}{2}$} & $\frac{5}{2}_{1}^{+} ; \frac{1}{2}$ (g.s.) & -1.09 & 2.24 & 1.11 & \\
\hline & $\frac{3}{2}_{1}^{+} ; \frac{1}{2}$ & 9.21 & 2.83 & 12.39 & \\
\hline & $\frac{7}{2}_{1}^{+} ; \frac{1}{2}$ & 7.76 & 3.83 & 11.23 & \\
\hline & $\frac{5}{2}_{2}^{+} ; \frac{1}{2}$ & -3.17 & -2.52 & -5.58 & \\
\hline $283^{+} ; 1$ & $2_{1}^{+} ; 0$ & 0.61 & 10.85 & 11.54 & $-5 \pm 1$ \\
\hline $31 \frac{5}{2}^{+} ; \frac{5}{2}$ & $\frac{3}{2}{ }_{1}^{+} ; \frac{3}{2}$ (g.s.) & 8.88 & 6.72 & 16.27 & $33 \pm 18$ \\
\hline $35 \frac{3}{2}^{+} ; \frac{3}{2}$ & $\frac{3}{2}_{1}^{+} ; \frac{1}{2}$ (g.s.) & 1.82 & 3.13 & 4.96 & $18.4 \pm 20.0$ \\
\hline
\end{tabular}




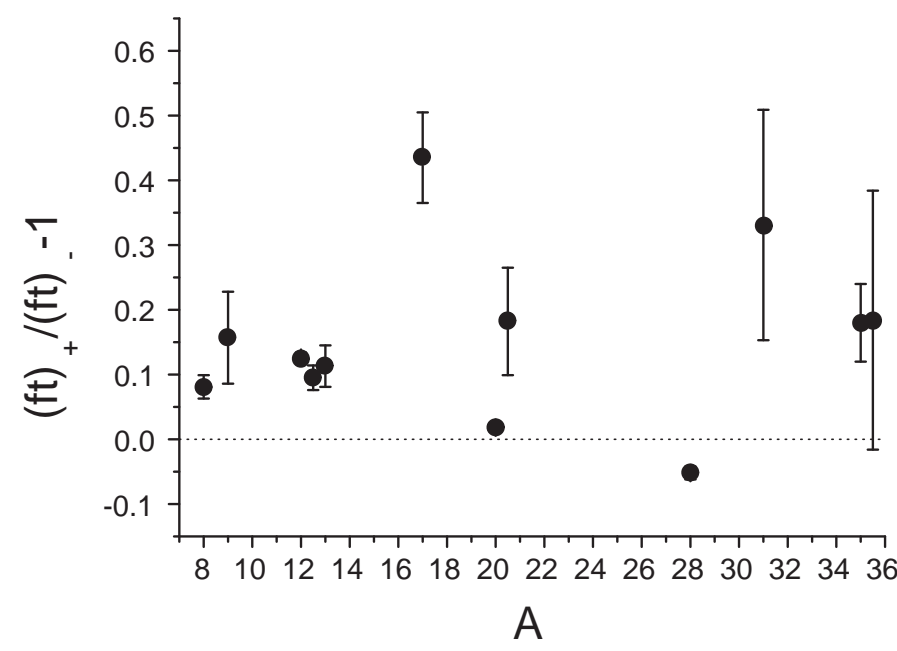

FIG. 1: Experimental asymmetry (4) deduced from the data on $\beta_{ \pm}$mirror transitions characterized by $|\Delta T|=1,|\Delta J|=0,1$, $\pi_{i}=\pi_{f}$ (calculated on the basis of the experimental $B(\mathrm{GT})$-values given in Tables II and III; see the captions of the tables for references). The results for the second transitions in $A=12, A=20 \mathrm{O}-\mathrm{F}-\mathrm{Mg}-\mathrm{Na}$ and $A=35 \mathrm{~S}-\mathrm{Cl}-\mathrm{K}-\mathrm{Ar}$ are shifted by 0.5 unit from the $A$-value.

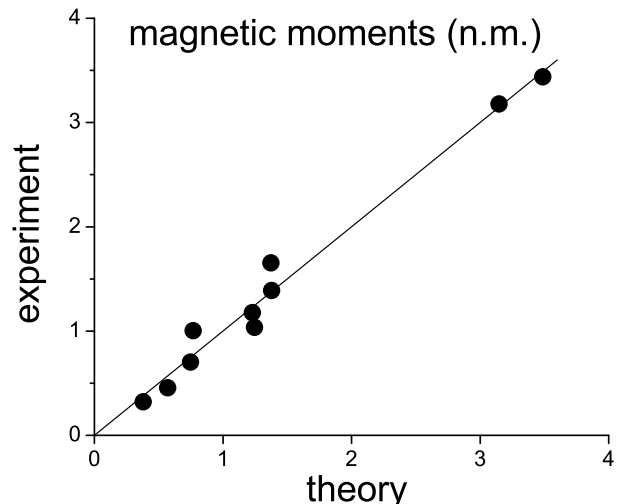

(a)

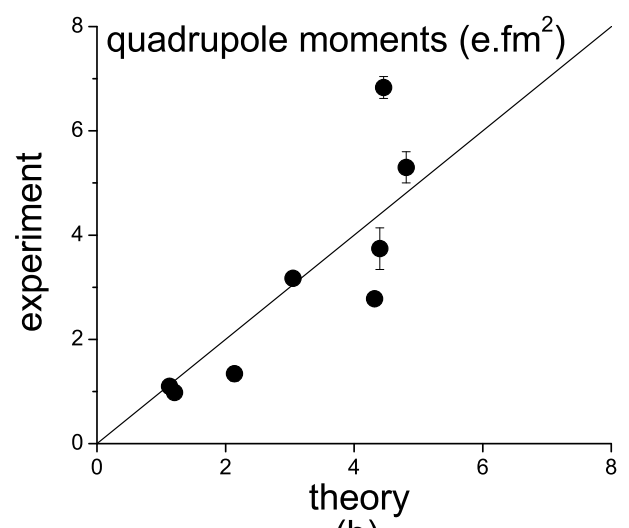

(b)

FIG. 2: Experimental versus theoretical (Table I): (a) absolute values of magnetic dipole moments of the ground states of $p$-shell nuclei obtained with the free-nucleon $g$-factors (data are taken from Ref. [46, 48, 50]); (b) absolute values of electric quadrupole moments of the ground states of $p$-shell nuclei obtained with the WS wave functions and $e(\pi)=1.35, e(\nu)=0.35$ (data are taken from Ref. [46, 47, 49, 51]). Solid lines correspond to the ratios equal to 1 and are given to guide the eye. 


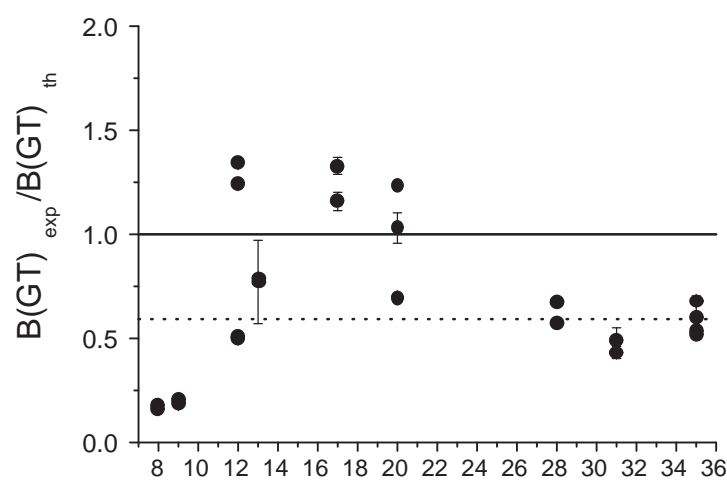

A

FIG. 3: Ratios of experimental to theoretical $B(\mathrm{GT})$ values present in Tables II and III (see the captions of the tables for references on the experimental data). Theoretical values are those obtained with the dependence on the parent state (column $(\mathrm{INC}+\mathrm{WS})^{*}$ in Tables II and III) and without dependence for $A=28,31$ and $A=35$ S-CL-K-Ar (column (INC+WS) in Table III) . Solid and dotted lines correspond to the rations 1 and $(0.77)^{2}$, respectively, and are given to guide the eye.
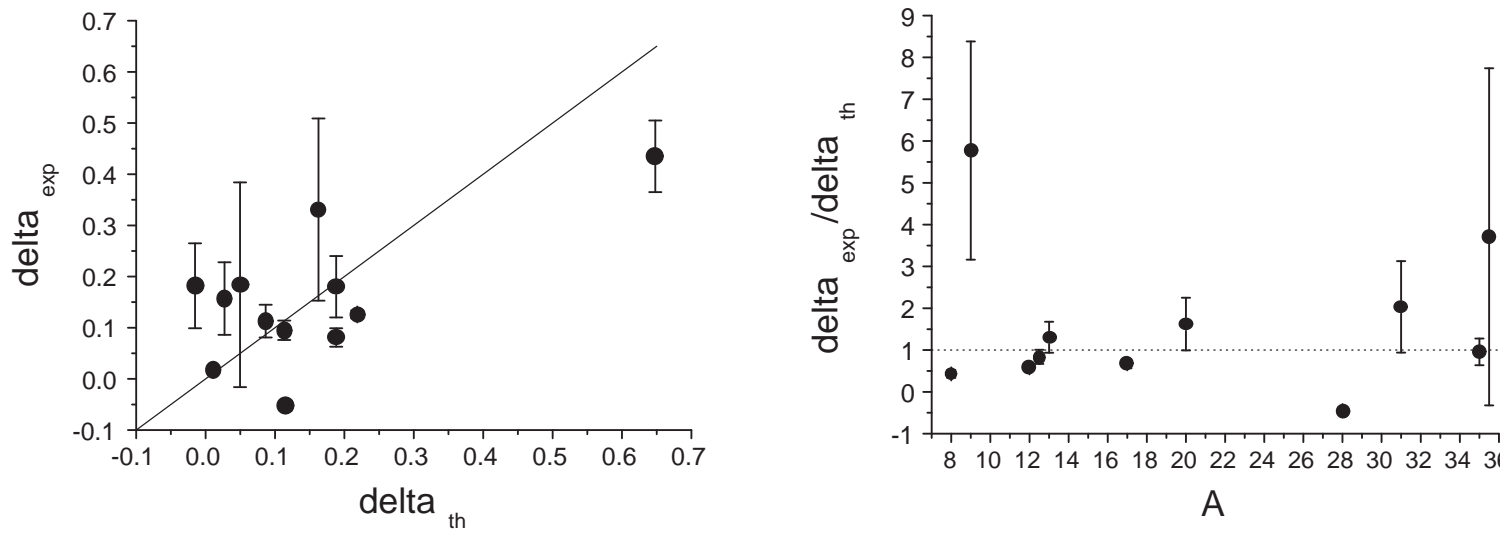

FIG. 4: Experimental versus theoretical asymmetries $\delta$ (left) and ratios of experimental to theoretical asymmetries (right) of selected GT decay rates for $p$ - and $s d$-shell nuclei. For a given mass, the theoretical points show the effect due to the inclusion of the dependence on the parent state, except for $A=28,31$ and $A=35$ S-Cl-K-Ar system. The results for the second transitions in $A=12$ and $A=35 \mathrm{~S}-\mathrm{Cl}-\mathrm{K}-\mathrm{Ar}$ are shifted by 0.5 unit from the $A$-value. The large in absolute value ratio of the asymmetries for $A=20$ case $\mathrm{O}-\mathrm{F}-\mathrm{Mg}-\mathrm{Na}$ is excluded. The experimental asymmetries are calculated on the basis of the experimental $B(\mathrm{GT})$-values from Tables II and III (see the captions of the tables for references). 